might be explained in terms of either a passive decay of stimulus traces or interference produced by intervening sounds (cf. Kinchla \& Smyzer, 1967: Wickelgren, 1969). These effects might be more clearly delineated by varying the interstimulus intervals and the number of choice sounds. The possible influence of decaying traces in particular would be clarified by determining whether incorrect choices of remote sounds tend to be sounds more intense than the sample. The significant interaction of tasks, intensities, and positions for $\mathrm{d}^{\prime}$ suggests that overall difficulty of detecting differences has some bearing on position effects, since the increased discriminability of sounds following the sample in A-S-BC and AB-S-C matching was much enhanced when intensity differences were large.

The significantly higher $\mathrm{H}$ rate for the left ear provides a further indication that dichotic presentation is not essential for the demonstration of ear asymmetry, and a further confirmation of right-hemisphere dominance for intensity discrimination (Doehring, 1972).

Although any further interpretation of the present findings must be highly speculative, the results do extend our knowledge of the conditions under which changes in auditory sequential processing occur. The previously observed variations in FA and $\mathrm{d}^{\prime}$ for S-ABC and ABC-S matching (Doehring, 1971; Doehring \& Libman, in press) were largely confirmed; and the two additional tasks revealed that the patterns of response bias and discriminability were consistently related to the temporal position of the sample sound. Further research is needed to determine whether nonverbal auditory sequences are indeed identified by a process analogous to that postulated by proponents of elementary association theories (cf. Bryden. 1967) rather than by a more dynamic and holistic process (cf. Lashley, 1951).

\section{REFERENCES}

Bryden, M. P. A model for the sequential organization of behavior. Canadian Journal of Psychology, 1967, 21, 37-56.

Doehring, D. G. Serial order effects in auditory discrimination by oddity and matching to sample. Perception \& Psychophysics, 1971, 10, 137-141.

Doehring, D. G. Ear asymmetry in the discrimination of monaural tonal sequences. Canadian Journal of Psychology, $1972,26,106-110$

Doehring, D. G., \& Libman, R. A. Signal detection analysis of auditory discrimination by children. Perceptual \& Motor Skills, in press.

Elliott, P. B. Tables of d'. In J. A. Swets (Ed.), Signal detection and recognition by human observers. New York: Wiley, 1964.

Green, D. M., \& Swets, J. A. Signal detection theory and psychophysics. New York: Wiley, 1966.

Guilford, J. P. Psychometric methods. (2nd ed.) New York: McGraw-Hill, 1954.

Kinchla, R. A., \& Smyzer, F. A diffusion model of perceptual memory. Perception \& Psychophysics, 1967, 2, 219-229.

Lashley, K. S. The problem of serial order in behavior. In L. A Jeffress (Ed.), Cerebral mechanisms in behavior. New York: Wiley, 1951

Wickelgren, W. A. Associative strength theory of recognition memory for pitch. Journal of Mathematical Psychology, 1969, $6,13-61$.

(Received for publication September 18, 1973.)

\title{
Stroop interference with long preexposures of the word: Comparison of pure and mixed preexposure sequences*
}

\section{FREDERICK N. DYER $\dagger$ \\ L.S. Army Medical Research Laboratory, Fort Knox, Ky. 40121}

The delay of color naming when the patches are incongruent color names was studied as a function of the duration of a preexposure of the word in black prior to coloration. Individual word and control stimuli were presented in a tachistoscope, and naming latencies were recorded with a voice key. Interference decreased to very low levels at $2 \mathrm{sec}$ of preexposure, then increased to intermediate levels for longer preexposures. Facilitation of color-naming by congruent color names generally paralleled interference effects. Sequences of stimuli in which the preexposure interval varied randomly from trial to trial showed longer color-naming times for short preexposures than did sequences where all stimuli in the sequence used the same preexposure. The utility of this and a related procedure for study of the central activity produced by viewing a written word was discussed.

Color-naming is substantially delayed when the color patches are shaped as words denoting colors different

*The author wishes to thank Dennis M. Buck, who collected the experimental data.

tRequests for reprints should be sent to Frederick N. Dyer, Experimental Psychology Division, U.S. Army Research Laboratory, Fort Knox, Ky. 40121. from the color of the patch (Stroop, 1935). This delay occurs despite instructions to ignore the word, and even extensive practice (Stroop, 1935; Jensen \& Rohwer, 1966) fails to eliminate this very reliable phenomenon. The delay of color-naming is a measure of an involuntary reading response, and provides a useful tool for study of written word stimuli (Dyer, 1973).

Dyer (1971) used the Stroop phenomenon to illustrate changes in the activity associated with a word stimulus during brief viewing periods. Individual words denoting colors were exposed in black for one of several short periods ( 0 to $500 \mathrm{msec}$ ), then the black word was illuminated in a color different from that denoted by the word. Ss were instructed to name the color as soon as possible following coloration of the black word. A series of Vs were exposed in black prior to coloration for a control condition. The difference in response latency between the incongruent name condition and the control Vs condition (Stroop interference) was found to first increase then decrease as a function of increasing duration of the black-word exposure prior to coloration. Black-word preexposures of 20,40 , and $60 \mathrm{msec}$ produced more delay of color-naming than a simultaneous exposure of the word and color ("0" 


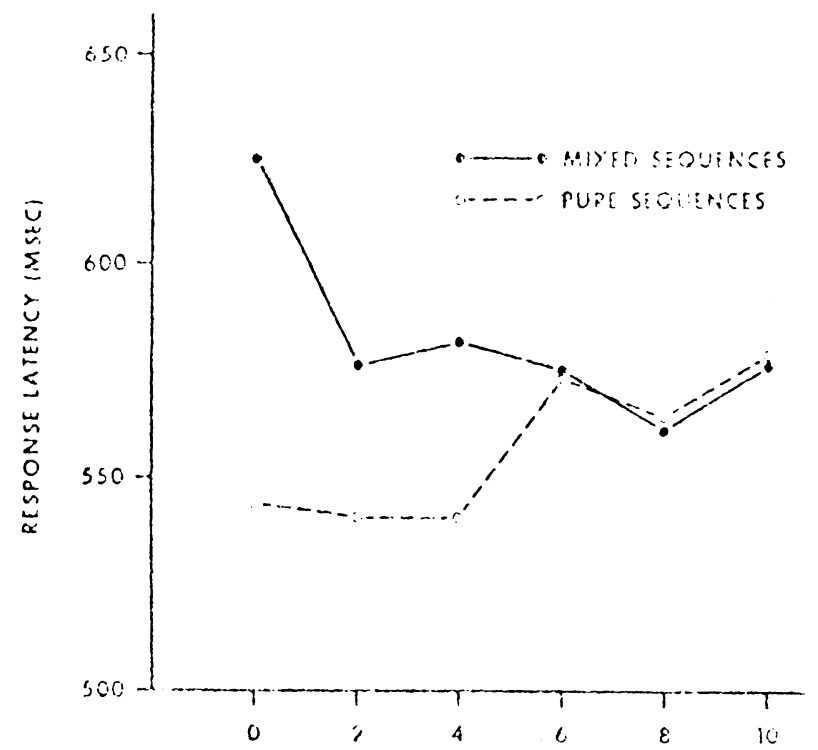

Fig. 1. Average response latency as a function of word preexposure duration for the mixed and pure stimulus sequences.

preexposure). Stroop interference then dropped sharply and was only a fraction of the earlier values with $200 \mathrm{msec}$ of black-word preexposure. However, even $500 \mathrm{msec}$ of black-word preexposure did not totally eliminate the word's capacity to delay naming of the color.

The primary purpose of the present study was to further explore changes in activity produced by word stimuli by measuring Stroop interference for longer exposures of the word in black prior to coloration. The question of whether any exposure would finally eliminate Stroop interference was of particular interest. A second purpose was to compare color-naming and interference for sequences of stimuli that always utilized the same preexposure duration (pure sequences) with color-naming and interference for sequences that randomly mixed the different preexposure durations (mixed sequences). Pilot work for the short-exposure study (Dyer, 1971) and an analogous experiment (Dyer, 1972) utilizing incongruent movement names as movement stimuli (with different stationary preexposures of the names prior to movement) indicated that mixed seuquences of preexposure durations produced different results than did pure sequences.

\section{METHOD}

\section{Subjects}

Twelve males, ranging in age from 17 to 21 years and with normal visual acuity and color vision, volunteered for the experiment.

\section{Apparatus and Stimuli}

Stimuli consisted of the words red. blue, and green and a series of Vs in the colors red, blue, and green. The words and Vs were cut out of white cards and presented in one field of a three-field tachistoscope (Scientific Prototype Model GB). Color filters over a white background were viewed through the cut-out letters with the illumination for the colors separate from that for the white cards. The illumination for colors was provided by a standard back-illuminator attached to the same field of the tachistoscope. The back-illuminator utilized the timers and circuitry normally used in one of the other fields of the tachistoscope. The words were centered in the exposure field and constructed of block capitals 3/8 in. high. Viewing distance was 50 in. Centered in the blank field was a small fixation cross on a white background. Luminance of the blank field and of the white background of the words was $8 \mathrm{fL}$. Color luminance were $3 \mathrm{fL}$ for red, $2 \mathrm{fL}$ for blue, and $5 \mathrm{fL}$ for green. Luminance was measured with a Spectra Brightness spot meter.

\section{Procedure}

After notification that the trial was ready, S fixated the cross, then pressed a switch which immediately exposed the word in black against a white background. After one of six preexposure intervals, the back-illuminator was turned on and the word appeared in one of the three colors against a white background. A millisecond clock began with coloration and was stopped by by a voice-operated relay when $\mathrm{S}$ gave his response. Preexposure intervals of $0,2,4,6,8$, and $10 \mathrm{sec}$ were used. Each $\mathrm{S}$ viewed the 12 different stimuli in each of two conditions. One used pure sequences, where all 12 stimuli were presented in random order at one preexposure interval. Four stimuli selected randomly from the 12 were presented prior to the 12 stimuli to familiarize $\mathrm{S}$ with the preexposure interval. Two such 16-stimulus sequences were presented in an experimental session. All six preexposures were completed at three successive sessions. The order of preexposure intervals was random, with each preexposure appearing in each position twice for the $12 \mathrm{Ss}$. Complementing the pure sequences was a mixed sequence condition, in which $\mathrm{S}$ received the 72 different preexposure-by-stimulus combinations in a completely random order (different for each $S$ ) at three sessions, which again involved two sequences of 16 stimuli. The first 4 stimuli at the beginning of each 16-stimulus sequence were warm-up stimuli for this mixed condition. Half of the Ss received the three pure sessions first followed by the three mixed sessions, and half received the mixed sessions followed by the pure sessions. Prior to any experimental sessions, the $S$ was acquainted with the six preexposures at one practice session. where 6 stimuli were presented at each of the six preexposure intervals. S was instructed to respond as quickly and accurately as possible. Feedback of the reaction times was given on each correct trial. Error stimuli were repeated later in the sequence to provide complete data. Sessions generally occurred on successive work days, and the time, including a 2 -min rest period between 16 -stimulus sequences, averaged about $20 \mathrm{~min}$.

\section{RESULTS}

The individual color-naming latencies were analyzed in an analysis of variance with one between-groups factor corresponding to whether Ss received pure or mixed sequences first, and with repeated measures on a two-level factor of sequences, a six-level factor of intervals, a three-level factor of colors, and a three-level factor of conditions. The three levels of the conditions factor corresponded to congruent combinations of words and colors, incongruent combinations, and control conditions. The basic data in the analysis were the mean of the two response latencies for incongruent combinations and the single latencies for each of the congruent and control combinations for a particular color, interval, sequence. and group. A highly significant main effect appeared for the conditions factor. $F(2.20)$ $=51.7 . \mathrm{p}<.001$, with mean color-naming latencies of 539. 565. and $605 \mathrm{msec}$ for the congruent, control. and 
incongruent combinations. respectively. A Newman-Keuls comparison of ordered means indicated all two-way comparisons of these three means to differ significantly $(p<.01)$. The nearly significant sequences main effect, $F(1,10)=4.80, p=.053$, reflected mean naming times of $557 \mathrm{msec}$ for pure sequences and $582 \mathrm{msec}$ for mixed sequences. The highly significant Sequence by Interval interaction, $F(5,50)=6.1$, $p<.001$, resuited from the occurrence of faster times for pure sequences for the three shorter preexposure intervals. Average response latencies for the two sequence types are plotted in Fig. 1 as a function of the preexposure interval.

Color produced a highly significant main effect, $F(2.20)=13.0, p<.001$, with an average naming time of $538 \mathrm{msec}$ for red, which was significantly different (Newman-Keuls, $\mathrm{p}<.01$ ) from the $586-$ and $585-\mathrm{msec}$ nar:ing times for blue and green, respectively. The intervals factor approached significance, $F(5.50)=2.18$, $\mathrm{p}<.10$, and produced a highly significant interaction with the conditions factor, $F(10,100)=5.01, p<.001$. This interaction is plotted in Fig. 2 and illustrates that interference drops sharply from an initially high level at the " 0 " exposure to a minimum at the $2-\mathrm{sec}$ exposure, and then rises to intermediate levels for the longer black preexposures. A similar change with intervals took place for the amount of facilitation of color-naming with congruent combinations. The groups factor main-effect means were nearly identical, and no interactions with this factor were significant.

\section{DISCUSSION}

The results of the present study show a large drop in interference from the " 0 " preexposure condition to the 2 -sec preexposure, and thus parallel findings of the earlier study (Dyer, 1971), which showed a sharp drop of interference from the identical " 0 " preexposure to a $1 / 2$-sec black-word preexposure. Although response latencies for the incongruent and control conditions were much higher for the mixed sequence condition than for pure sequences for these short preexposures, the difference between the two (Stroop interference) was quite similar for the two sequence conditions.

The rise of interference with longer exposures suggests that activity corresponding to the word stimulus may vary cyclically over time. Such activity would be relatively well synchronized near stimulus onset, producing the observed large changes in response latency for short preexposures. Variations from trial to trial in the frequency of such a waxing and waning would produce intermediate values of average interference for longer preexposure intervals.

It should be noted that congruent combinations serve an important methodological function. There is an opportunity for $S$ to restrict his response set to colors incongruent to the preexposed color name if they are not included. Since for " 0 "-duration preexposures (and other short preexposures) there is much less chance to reduce the response set in this manner, the differences between different preexposure conditions could reflect this difference in response strategy rather than changes in activity associated with the word. Facilitation differences as a function of preexposure interval generally paralleled the changes in the amount of interference, and both may be indices of central word activity.

Whereas the present paradigm allows study of changes in central word activity as a function of the length of time of

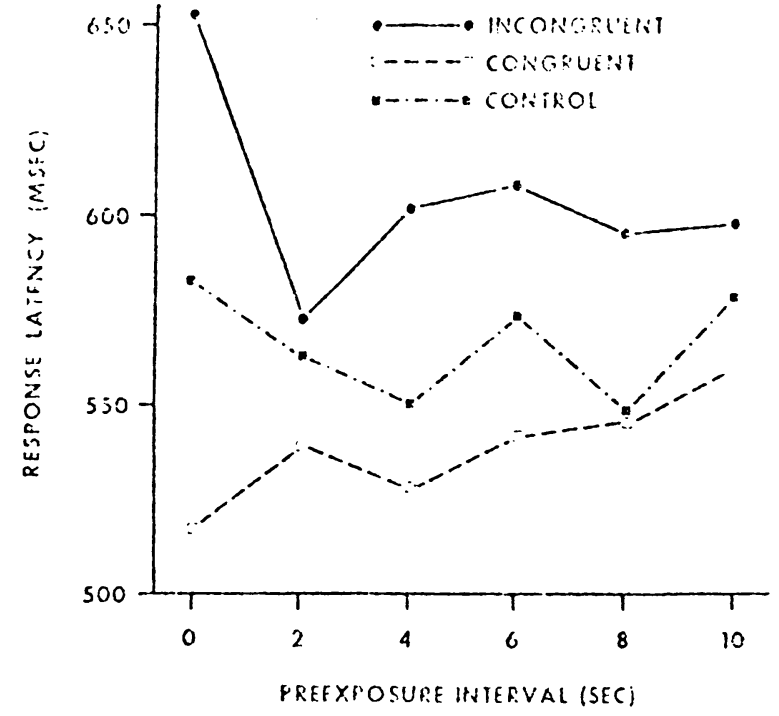

Fig. 2. Average response latency as a function of word preexposure duration for the congruent, incongruent, and control conditions.

continuous viewing of a word, another related procedure permits study of changes in central word activity as a function of the length of elapsed time following a brief exposure of a word. Dyer \& Severance (1973) used separate black words and nonword color patches as stimuli and presented these successively in a tachistoscope. A highly reliable delay of color-naming occurred when the word was incongruent to the color that followed it, and a less pronounced facilitation was found when the word and color were congruent. Four intervals between word offset and color onset were used in the study $(0$, $25,50,100 \mathrm{msec}$ ), and all produced about the same amount of interference to color-naming. Longer intervals between the word and color would have surely reduced the interference, and the interval that marked this drop would provide an index of the duration of the central activity following the discrete presentation of the written word. This successive word-color paradigm would also allow study of the effects of interposing "masking" stimuli between the word and color. Such "masking" could be of the traditional type that interferes with visual perception (Eriksen \& Eriksen, 1971). However, it might be even more interesting to allow unrestricted perception of the word and then find what stimuli, if any, will "mask" the word's "meaning."

\section{REFERENCES}

Dyer, F. N. The duration of word meaning responses: Stroop interference for different preexposures of the word. Psychonomic Science, 1971, 25, 229-231.

Dyer, F. N. Latencies for movement naming with congruent and incongruent word stimuli. Perception \& Psychophysics, 1972, $11,377-380$.

Dyer, F. N. The Stroop phenomenon and its use in the study of perceptual, cognitive, and response processes. Memory \& Cognition, 1973, 1, 106-120.

Dyer, F. N., \& Severance, L. J. Stroop interference with successive presentations of separate incongruent words and colors. Journal of Experimental Psychology, 1973, 98, 438-439.

Eriksen, C. W., \& Eriksen, B. A. Visual perceptual processing rates and backward and forward masking. Journal of Experimental Psychology, 1971, 89, 306-313.

Jensen, A. R., \& Rohwer, W. D., Jr. The Stroop color-word test: A review. Acta Psychologica, 1966, 25, 36-93.

Stroop, J. R. Studies of interference in serial verbal reactions. Journal of Experimental Psychology, 1935, 18, 643-662.

(Received for publication September 14, 1973.) 